Facilitation of word recognition by semantic priming in schizophrenia

By: Thomas R. Kwapil, Douglas C. Hegley, Loren J. Chapman and Jean P. Chapman

Kwapil, T.R., Hegley, D.C., Chapman, L.J., \& Chapman, J.P. (1990). Facilitation of word recognition by semantic priming in schizophrenia. Journal of Abnormal Psychology, 99, 215221.

Made available courtesy of the American Psychological Association: http://www.apa.org/

***Reprinted with permission. No further reproduction is authorized without written permission from American Psychological Association. This article may not exactly replicate the final version published in the APA journal. It is not the copy of record. ***

\begin{abstract}
:
Schizophrenic $(\mathrm{n}=21)$, bipolar $(\mathrm{n}=18)$, and normal control subjects $(\mathrm{n}=21)$ were compared on a word recognition measure of semantic priming. The task involved the presentation of related, neutral, and unrelated word pairs; the second word (target word) in each pair was presented in a degraded form. Facilitation was defined as the accuracy of target word recognition for the related word pairs minus accuracy for the neutral word pairs. Titration, achieved by manipulating the degradation of the target word, was used to maintain each subject's overall accuracy for related and neutral items at approximately 50\%. This procedure minimized the artifactual effects of overall accuracy on the difference score. Schizophrenics exceeded both normal control subjects and bipolar subjects on facilitation. Bipolar subjects did not differ from control subjects. The results support Maher's hypothesis that semantic priming effects are heightened in schizophrenia.
\end{abstract}

Keywords: bipolar disorder | priming | schizophrenia | semantics | word recognition | stimulus parameters | semantic priming | psychology

Article:

Acknowledgement: Preparation of this article was supported in part by National Institute of Mental Health Grant MH39469 and Research Scientist Award MH00743, both to Loren J. Chapman. 
We thank Laurie A. Frost and Michael B. Miller for their comments and suggestions on this research. We thank the directors of the Off the Square Club, Marcus McCoy, and Kathleen Reilly, as well as all of the members and employees of the club for their generous cooperation.

Douglas Hegley is now at Adelphi University.

Correspondence concerning this article should be addressed to: Thomas R. Kwapil, Department of Psychology, University of North Carolina-Greensboro, 296 Eberhart Building, Greensboro, North Carolina 27412-5001

Schizophrenic discourse is often characterized by associative intrusions in which strong associates to earlier words supplant contextually appropriate speech. Bleuler (1911/1950) provided the famous example of his patient who listed her family members as "father, son ... and the Holy Ghost” (p. 26). The present study examines heightened intrusion of activated associations in the responses of schizophrenics. A semantic priming task scored for response accuracy is used to measure the effects of activated associations and to study group differences between schizophrenic and normal control subjects. The study also examines the performance of bipolar patients to provide an indication of whether other psychopathological patients resemble schizophrenics in their semantic priming performance.

Maher (1983) explained the associative intrusions in schizophrenic speech in terms of heightened effects of semantic priming. According to the two-process theory of Posner and Snyder (1975), the activated associations of normal individuals decay rapidly or are inhibited, which limits their potential for intrusion into discourse. Schizophrenics, Maher suggested, may have a defect in either the decay or the inhibitory process, which results in the intrusion of activated associations into their speech.

Maher's suggestion was built in part on the findings of Meyer and Schvaneveldt (1971) and Schvaneveldt and Meyer (1973), who measured associatively based semantic priming on a lexical decision task. They found that normal subjects were able to identify a target stimulus (e.g., NURSE) as a word rather than as a nonword more rapidly when it was immediately preceded by an associatively related priming word (e.g., DOCTOR) than when it was preceded by an unrelated word (e.g., BREAD). This effect is known as facilitation by semantic priming. Schvaneveldt and Meyer explained their results in terms of an automatic spreading of activation from a word to its associates. Because schizophrenics frequently exhibit associative intrusions in their speech, Maher (1983) proposed that they would exceed normal subjects on the effects of associatively based semantic priming on a laboratory measure. This hypothesis is neutral as to the several alternative mechanisms of heightened priming effects. Such effects could reflect 
stronger priming, delayed or more prolonged priming, or a defect in the inhibition of associates, so that the associates remain activated when the target word appears.

According to de Groot, Thomassen, and Hudson (1982), the unrelated condition is not an appropriate baseline against which to assess the effects of semantic priming because the priming word initiates the processing of related words, thereby inhibiting the processing of the unrelated target word. Thus, the use of the unrelated items as a control condition appears to underestimate the baseline and overestimate facilitation by associative priming. Antos (1979) and de Groot et al. (1982) suggested that the responses to target words preceded by repetitively presented neutral priming words, such as BLANK or NEUTRAL, serve as more appropriate baseline measures because such words do not initiate processes that influence subsequent target processing. The use of the neutral condition is intended to separate the effects of facilitation, produced by the related priming word, and inhibition, produced by the unrelated priming word.

Posner and Snyder (1975) contended that inhibition is the result of strategic or attentional processes and does not involve automatic spreading activation. More recent evidence, however, has indicated that spreading activation may play some role in inhibition, but less than in facilitation ( de Groot, Thomassen, \& Hudson, 1986; Neely, 1977). The hypothesis of the present research is one of heightened effects of the automatic spread of activation, not of strategic effects. Schizophrenics are commonly described as poor at strategic allocation of attentional resources. Therefore, it is important to attempt to minimize the opportunity for facilitation by strategic deployment of attention in the measurement of facilitation.

Previous investigations of semantic priming in schizophrenic subjects have provided tentative support for the hypothesis of increased facilitation in schizophrenia. Onifer (1980) presented subjects with a sentence (aurally) and a letter string (visually) and instructed them to report whether the letter string was a word or a nonword. When the letter string was a word, it appeared either concurrently with a related priming word in the sentence or after a brief delay. Onifer interpreted his data as indicating that schizophrenics exhibited greater semantic priming than normal subjects. Onifer's data, however, were only partially supportive of that conclusion and were based upon a small number of subjects and items.

Manschreck et al. (1988) compared the performance of thought-disordered schizophrenics $(\mathrm{n}=$ 12) , non-thought-disordered schizophrenics $(n=6)$, unipolar affective disorder patients $(n=$ 9 ), and normal control subjects $(n=11)$ on a lexical decision task. Semantic priming effects were measured by comparing response latency on related items to latency on unrelated items. The investigators found an overall difference among the groups on semantic priming. However, they did not report posterior comparisons between the pairs of groups, so it is not possible to determine whether either schizophrenic group, or both combined, differed from either the normal control subjects or the unipolar patients. Therefore, their findings must be regarded as only suggestive. In addition, their experimental design did not allow the investigators to separate the effects of facilitation and inhibition in their measure of priming, since the recognition of related 
target words would be facilitated and the recognition of unrelated target words would be inhibited. Manschreck et al. also found that the schizophrenic subjects tended (nonsignificantly) to have generally shorter response latencies than the normal subjects. This result is surprising given the common finding that schizophrenics tend to respond slower than normals on most tasks ( Nuechterlein, 1977). Although the lack of reported differences between the pairs of groups might be viewed as discouraging to the hypothesis of heightened effects of priming in schizophrenia, we suggest that it need not be so viewed because the results were based upon a small number of subjects (6 to 12 subjects per group) and items (10 related and 10 unrelated items).

\section{Methodological Considerations in the Design of the Task}

Semantic priming effects are measured, either implicitly or explicitly, as the difference score between a pair of response latencies or a pair of accuracy scores. However, in either case, a serious potential artifact arises in the measurement of the difference score. This artifact affects the study of both individual and group differences. In the case of response latency, which is generally the focus of interest in the lexical decision task, the latency difference might be expected to be greater for subjects with greater generalized slowness as indexed by the sum of the two latency components. Sources of difficulty more often interact instead of acting additively. For example, a group of slow responders whose mean latencies are twice as long as those of normal subjects on each task will show twice the difference between the two mean latencies. Because schizophrenics are slower than normals on most tasks, they may artifactually show a greater difference between two latency measures and, hence, greater priming effects.

The difference score of accuracy, just as of latency, is artifactually related to the sum of the scores. Chapman and Chapman $(1988,1989)$ presented a detailed discussion of this artifact and the problems that it presents for measuring group and individual differences in cognitive deficit. They demonstrated that for any two dichotomously scored free-response tasks, A and B, that are scored for accuracy, there is a curvilinear relationship between the $(A-B)$ difference score and the $(A+B)$ score of overall accuracy, assuming that guessing makes only a minimal contribution to accuracy. The $(A-B)$ difference score is greatest when the $(A+B)$ overall accuracy is at $50 \%$ (the point of maximal discriminating power for such tasks), and is attenuated as $(\mathrm{A}+\mathrm{B})$ overall accuracy departs from $50 \%$ in either direction. Therefore, larger $(A-B)$ difference scores will tend to be found for subjects with $(A+B)$ overall accuracies near $50 \%$, and smaller $(A-B)$ difference scores will tend to be found for subjects with $(A+B)$ overall accuracies that are either larger or smaller than 50\%. This relationship follows directly from the relationship between task variance and task difficulty.

Chapman and Chapman $(1988,1989)$ suggested that one method for avoiding this artifact is to titrate each subject's $(A+B)$ overall accuracy to a predetermined level by manipulating another variable that affects $(A+B)$ accuracy, but does not independently affect the $(A-B)$ difference 
score. The use of the term titration in this article refers to the maintenance of a subject's $(A+B)$ overall accuracy at a constant level.

The present study was designed to measure semantic priming using accuracy of identification of the target word rather than response latency as the dependent variable. While accuracy is measured in the lexical decision task, the level of accuracy is usually too high to be discriminating as a dependent variable in its own right. It is measured primarily to ensure that the results cannot be attributed to a speed-accuracy trade-off. Accuracy, in the present study, was maintained in a middle range, where the accuracy score is more discriminating. Facilitation was measured as the difference score between the number of correct recognitions of target words that were preceded by a related priming word minus the number of correct recognitions of target words that were preceded by a neutral priming word.

In the present study, the overall accuracy on related (R) and neutral $(\mathrm{N})$ items of each subject was titrated to $50 \%$ by degrading the presentation of the target words through the deactivation of a portion of the pixels of light that composed the words on the computer screen. The titration procedure ensured that each subject's $(\mathrm{R}-\mathrm{N})$ difference score measure of facilitation would not be differentially constrained by the subject's $(\mathrm{R}+\mathrm{N})$ overall accuracy, because each subject's $(\mathrm{R}$ $+\mathrm{N}$ ) overall accuracy was maintained at a constant level.

The constant $(\mathrm{R}+\mathrm{N})$ accuracy level must be maintained successfully throughout the series of items rather than as an average of high-accuracy blocks of items and low-accuracy blocks. A mixture of high- and low-accuracy blocks would artifactually constrain the $(\mathrm{R}-\mathrm{N})$ facilitation score just as if the $(\mathrm{R}+\mathrm{N})$ overall accuracy score were very high or very low throughout all the items. Therefore, the present study used frequent adjustment of the level of target word degradation to maintain each subject's $(\mathrm{R}+\mathrm{N})$ accuracy close to $50 \%$ throughout the entire range of items.

The related, neutral, and unrelated items were presented to subjects intermixed in blocks, and the subject's $(\mathrm{R}+\mathrm{N})$ accuracy was assessed after every block. If a subject's $(\mathrm{R}+\mathrm{N})$ accuracy was greater than 50\%, the level of degradation was increased for the following block of items, thereby tending to lower the $(\mathrm{R}+\mathrm{N})$ accuracy. Likewise, if $(\mathrm{R}+\mathrm{N})$ accuracy was less than $50 \%$, the level of degradation was decreased for the subsequent block of items. This titration procedure removes individual differences in overall accuracy as well as practice and fatigue effects across trials.

The titration design has an unconventional feature that may initially seem counterintuitive. If ( $\mathrm{R}$ $+\mathrm{N}$ ) overall accuracy is successfully titrated to $50 \%$, a subject who scores above $50 \%$ on the related items must score below $50 \%$ on the neutral items by a similar amount. Therefore, using the titration procedure, the absolute levels of performance on the related and neutral items are not meaningful or interpretable; rather the difference score is meaningful. Conversely, in the usual nontitration design, the $(\mathrm{R}-\mathrm{N})$ difference score measure of facilitation is distorted by the artifact 
when subjects differ widely in $(\mathrm{R}+\mathrm{N})$ overall accuracy, although the related and neutral component scores are not affected by the artifact. The titration procedure used in the present study does not completely eliminate variations in $(R+N)$ overall accuracy, because titration is usually imperfect. The procedure does, however, sharply reduce such variations and thus minimizes the effect of the artifact on the facilitation score.

The reader might be inclined to suggest that instead of titrating $(\mathrm{R}+\mathrm{N})$ overall accuracy to $50 \%$, one might instead titrate the accuracy of the neutral items to $50 \%$. Then, it might seem, one could simply compare component $(\mathrm{R})$ scores for subjects who have equivalent $(\mathrm{N})$ scores. Given this procedure, the accuracy on the neutral items would be $50 \%$, the level of greatest task reliability and discriminating power. However, accuracy on the related items would deviate from $50 \%$ and thus would be less reliable and discriminating. Furthermore, the subjects who have the greatest propensity toward priming would be farthest away from $50 \%(\mathrm{R}+\mathrm{N})$ overall accuracy, so that their $(\mathrm{R}-\mathrm{N})$ priming score would be limited or reduced by the artifactual relationship of the difference score to accuracy more than would the scores of subjects who produced less priming. As a result, differences in priming among subjects would tend to be obscured.

Measurement of Inhibition

The present design also yields a measure of inhibition in processing semantically unrelated items. Inhibition is measured as the difference score between the number of correct recognitions of target words that are preceded by a neutral priming word minus the number of correct recognitions of target words that are preceded by an unrelated priming word. Because strategic allocation of attention is thought to play a major role in inhibition ( de Groot et al., 1986; Neely, 1977) and schizophrenics are considered to be deficient at strategic allocation ( Gjerde, 1983; Nuechterlein \& Dawson, 1984), we do not offer a prediction concerning any defects of inhibition in the schizophrenics. The neutral minus unrelated $(\mathrm{N}-\mathrm{U})$ difference scores are biased by variations among subjects in $(\mathrm{N}+\mathrm{U})$ overall accuracy. Therefore, interpretations of the findings concerning inhibition must take the effects of the artifact into consideration. Accuracy on the unrelated items could not be included as a factor in titration. Although the relationship between the $(\mathrm{A}+\mathrm{B})$ overall accuracy score and the $(\mathrm{A}-\mathrm{B})$ difference score is known for any two variables, comparable information is not available for three variables. Because our specific predictions involved the difference between the related and neutral items, we chose to control overall accuracy for those two variables alone.

Method

Subjects

Twenty-one schizophrenic outpatients (15 males, 6 females), 18 bipolar outpatients (11 males, 7 females), and 21 normal control subjects (15 males, 6 females) participated in the study. Schizophrenic and bipolar subjects were recruited and tested at a Madison, Wisconsin, day “club” for previously hospitalized psychiatric patients. This facility provides a noontime meal, 
recreational activities, and housing and occupational placement services. Since all of the club's services are provided during the day, the members are typically not gainfully employed.

Fifteen of the schizophrenic subjects and 14 of the bipolar subjects were receiving psychotropic medication at the time of the study. Eleven of the schizophrenic subjects were taking fluphenazine decanoate, while 1 each was taking trifluoperazine, chlorpromazine, thiothixene, and haloperidol. Eight of the schizophrenic subjects receiving neuroleptics were also taking anticholinergics (7 were taking benzotropine mesylate, while 1 was taking trihexyphenidyl). Twelve of the bipolar subjects were receiving lithium carbonate, while 2 were receiving carbamazepine. Of the bipolar subjects on lithium carbonate, 4 were also receiving neuroleptics (3 on fluphenazine decanoate, and 1 on thiothixene), and 1 each was taking imipramine, amitriptyline, and phenytoin. One of the bipolar subjects on fluphenazine decanoate was also taking benzotropine mesylate.

Diagnoses were made according to the Diagnostic and Statistical Manual of Mental Disorders (3rd ed.; American Psychiatric Association, 1980) criteria after interviewing the subjects and examining their clinical records. Fourteen of the bipolar subjects had a history of psychotic symptoms. Students from a local technical school and individuals solicited from the community were used as a control group. All subjects were paid for their participation. The groups did not differ on mean years of age: schizophrenics, 33.9 ( $\mathrm{SD}=8.3$ ), bipolars, 34.0 ( $\mathrm{SD}=8.6$ ), and controls, 32.7 ( $\mathrm{SD}=6.8)$; or mean years of education: schizophrenics, $12.2(\mathrm{SD}=1.4)$, bipolars, 12.1 ( $\mathrm{SD}=1.7)$, and controls, $12.9(\mathrm{SD}=1.7)$.

Apparatus

Stimulus presentation and data collection were controlled by a Compaq (Houston, TX) portable computer, model \#101709, interfaced with a rapid decay Taxan (Industry, CA) 620 color video monitor. All words were presented on the video screen in uppercase standard IBM color graphics adapter font and were $9 \mathrm{~mm}$ in height. At any given degradation level, a particular letter always had the same pixels deactivated regardless of the word in which the letter appeared. Furthermore, a particular letter at a given level of degradation always had the same pixels deactivated as at any lower level, along with additional deactivated pixels. These precautions were used in an attempt to ensure that accuracy of recognizing any given word declined with increased degradation. The computer program enabled the experimenter to vary the percentage of pixels deactivated in $2 \%$ increments.

\section{Materials}

Each item consisted of a sequence of two words: a nondegraded initial word followed by a degraded target word. Related items contained the priming word followed by a related target word: for example, BELLS (nondegraded), CHIME (degraded). Neutral items contained the word BLANK followed by a target word: for example, BLANK (nondegraded), CHIME 
(degraded). Unrelated items contained an initial word followed by an unrelated target word: for example, STRAW (nondegraded), CHIME (degraded).

The neutral condition was included to provide a baseline measure against which to assess the effects of semantic priming in accordance with the suggestions of Antos (1979) and de Groot et al. (1982). Jonides and Mack (1984) reviewed various potential problems with the use of alternative neutral conditions. A principal disadvantage of using a neutral word such as BLANK is that its repeated presentation may induce less attention or alertness to the neutral items than does the use of a new priming word in each related item. However, the use of a single neutral word seems the best available alternative. Following the advice of Jonides and Mack (1984), we matched the nonneutral priming words with BLANK on word length and mean word frequency.

The unrelated condition was included primarily to provide variety in the relationships of the first and second words. If all words other than BLANK were followed by a related word, unwanted strategic processes would be facilitated. The prospective unrelated items were evaluated by eight judges for the presence of any association between the priming and target words to ensure that they did not contain either a direct or a mediated relationship. Furthermore, the prospective target words were evaluated by the judges to ensure that they were not related to the neutral priming word BLANK.

To maintain the $(\mathrm{R}+\mathrm{N})$ overall accuracy of the subjects at a constant level, it is necessary to use items within each task that are similar in difficulty when they are nondegraded. Otherwise, adjustments in difficulty that are made for one block of items will be inappropriate for another block. Therefore, several potential sources of variability in difficulty among items were kept within a narrow range as well as equated across the conditions. Previous investigators ( Balota \& Chumbley, 1984; Riegel \& Riegel, 1961) demonstrated that high-frequency target words are more readily identifiable than low-frequency target words. Thus, only words with a relatively low derived frequency were considered for use in the present study. Word frequency values were obtained from Kucera and Francis (1967) and refer to incidence per 1 million words. We used a derived frequency measure, obtained by adding $75 \%$ of the frequency of the stimulus word to $25 \%$ of its plural form (in the case of singular words) or singular form (in the case of plural words). All priming and target words in the present study were comprised of five letters and one syllable.

A pool of 240 related word pairs was generated that met the previously mentioned criteria. The word pairs were presented to 70 undergraduate students who rated the pairs on associative strength. The subjects were instructed to rate on a 5-point scale "the extent to which the first word tends to call the second word to mind.” On the basis of these ratings, the pool of related word pairs was reduced to 96 items. Mean associative strength was 3.43 (range: 2.23-4.84). An example of an item pair of lower associative strength is SKIRT-GOWNS (2.26), while an example of a pair of stronger associative strength is THIEF-CROOK (4.11). Stronger associates were omitted to avoid the unwanted strategic effect of the subject being able to anticipate the 
appearance of a particular target word. The mean derived frequency of the target words in this study was 4.78 (range: 0.00-21.00). The mean derived frequency of the nonneutral priming words was 12.29 (range: 0.00-44.00). The derived frequency of the neutral priming word BLANK was 11.25. An example of a low-frequency word is STALK (0.00), while an example of a higher frequency word is BEARD (20.75). The 96 related items were divided into three sets, matched on their distribution of associative strength and target word frequency. The three sets of target words were used to generate three lists of items; each list contained one third of each of the three kinds of items intermixed. To avoid the effects of graphemic and phonemic priming, related and unrelated word pairs shared no more than one letter in the same position and never shared the first letter.

A counterbalanced design was used in which each target word appeared in a different type of item (related, neutral, or unrelated) on each list. For example, the related target words from list 1 were used as neutral target words on list 2 and as unrelated target words on list 3. Each priming and target word, with the exception of the neutral word BLANK, appeared only once on each list. Each of the three lists was given to one third of the subjects in each of the three groups. The method of counterbalancing target words so that every target word appeared as each of the three types of items was used to match perfectly the three types of items on characteristics of the target words. As a result of this design, however, subjects within the same group received different lists, making it impossible to examine individual differences on facilitation.

The items in each list were ordered in blocks of three for the purpose of titration. Each block contained a related item, a neutral item, and an unrelated item. The subjects, however, were not able to develop expectations concerning the ordering of related, neutral, and unrelated items because the items were randomly assigned within each block and the blocks were not apparent to the subjects. Twenty-four practice items (eight from each condition) were constructed and presented before the experimental items.

\section{Procedure}

Subjects were tested individually in a darkened, sound-protected room, and were seated $90 \mathrm{~cm}$ from a video screen that was elevated to eye level. The experimenter sat at the computer terminal to the right of the subject's video screen, partially obscured from the subject. The experimenter viewed a separate computer monitor that provided the same information that appeared on the subject's video screen, as well as information about the current level of degradation and prompts to enter the subject's accuracy on the previous trial.

Subjects were informed that they would be presented with a series of trials, each of which contained a nondegraded word followed by a degraded word. The subjects were also told about the three types of items and were shown examples of each printed on a piece of paper. They were instructed to name both words as accurately and as quickly as possible when the degraded word appeared. The subjects were required to name the priming word to ensure that they attended to it. 
The subjects were reminded during the presentation of the instructions and the practice trials to respond loudly and clearly on each trial so that the experimenter could score the trial as correct or incorrect. Response latencies to the target words could not be accurately measured because the subjects were instructed to name both words. While the subjects were instructed to respond both quickly and accurately, we believe that our results are not attributable to a speed-accuracy tradeoff. Our review of the literature supports Massaro, Jones, Lipscomb, and Scholz' (1978, p. 506) report that there is generally a positive correlation between error rate and response latency in word-naming tasks. Therefore, our paradigm should not produce a speed-accuracy trade-off.

The practice trials were used to obtain an appropriate initial degradation level and to familiarize the subjects with the task. The sequence for both the practice and the experimental trials was as follows: (a) a tone from the computer for $250 \mathrm{~ms}$; (b) a row of three asterisks separated by one space from one another $(* * *)$ in the exact location of the upcoming words for $418 \mathrm{~ms}$; (c) a blank screen for $582 \mathrm{~ms}$; (d) the nondegraded priming word for $418 \mathrm{~ms}$; (e) a blank screen for 82 ms; (f) the degraded target word for $985 \mathrm{~ms}$; (g) a blank screen for 1,015 ms; and (h) a final tone from the computer signaling the end of the trial. These display times were selected because they produced reliable priming effects in pilot studies with normal control subjects.

In keeping with the recommendation of Reed (1979), the vertical synchronization pulse timing signal was connected to the sense line that activated the software to enhance the accuracy of the timing. Timing of the stimulus presentation is dependent upon the fact that the beam refreshes the computer screen (completes a vertical retrace) every 1/60 of a second, starting at the top of the screen. While the stimulus presentation appears continuous, most of the stimulus decays in roughly $2 \mathrm{~ms}$ following each vertical retrace. Presentation time as reported here is the number of vertical retraces multiplied by $16.67 \mathrm{~ms}$, less $15 \mathrm{~ms}$, rounded to the nearest millisecond. The blank screen time reported is the number of vertical retraces omitted plus $15 \mathrm{~ms}$.

Responses were scored as correct or incorrect. Alternative forms of the correct words, such as changes in tense or from singular to plural, were scored as incorrect. While reliability data on scoring were not available, the assessment of whether a subject provided a correct or incorrect response was a simple process and was unlikely to have varied as a function of condition or group. Trials on which the subjects did not provide a response or did so after the final tone were scored as incorrect. Following each block of three trials, the computer determined the subject's mean accuracy on the preceding two neutral and two related trials and then adjusted the degradation level for the next block of trials by the following rules: four responses correct, $+4 \%$; three correct responses, $+2 \%$; two correct responses, maintain current level; one correct response, $-2 \%$, no correct responses, $-4 \%$. Performance on the unrelated trials was not considered in the titration procedure.

The initial degradation level on the experimental trials was determined individually for each subject during the practice trials. Each subject began the practice trials at $20 \%$ degradation. The 
degradation level was increased during the practice trials until the subject performed at 50\% ( + N) accuracy.

Ninety-six experimental trials were presented to each subject. Two-minute rest intervals followed trials 33 and 66. Subjects were also informed that they could request a brief rest period at any time during the course of the session. Two bipolar, 4 schizophrenic, and 1 control subject did so. Following the test session, the subjects were debriefed as to the nature of the semantic priming task. However, none of the control subjects were informed about comparisons with clinical groups, and none of the subjects were informed about the specific hypothesis of greater facilitation by schizophrenics.

Results

Table 1 presents the mean percentages of correct scores for each condition as well as the facilitation and inhibition scores for each group. Facilitation was measured by subtracting the neutral score from the related score for each subject. Inhibition was measured by subtracting the unrelated score from the neutral score.

Table 1

Mean Percentages for Correct Responses and for Facilitation and Inhibition Scores

\begin{tabular}{|c|c|c|c|c|c|c|}
\hline \multirow[b]{2}{*}{ Score } & \multicolumn{2}{|c|}{ Schizophrenics $(n=21)$} & \multicolumn{2}{|c|}{ Bipolars $(n=18)$} & \multicolumn{2}{|c|}{ Controls $(n=21)$} \\
\hline & $M$ & $S D$ & $M$ & $S D$ & $M$ & $S D$ \\
\hline Related & 61.4 & 5.6 & 57.5 & 5.3 & 55.4 & 7.1 \\
\hline Neutral & 43.0 & 4.1 & 48.1 & 5.4 & 48.1 & 5.8 \\
\hline Unrelated & 34.7 & 8.0 & 45.7 & 7.6 & 41.7 & 9.5 \\
\hline Facilitation & 18.4 & 5.6 & 9.4 & 9.9 & 7.3 & 12.2 \\
\hline Inhibition & 8.3 & 8.9 & 2.4 & 9.0 & 6.4 & 9.2 \\
\hline
\end{tabular}

Note. Facilitation scores are related percentage correct minus neutral percentage correct, and inhibition scores are neutral percentage correct minus unrelated percentage correct.

The titration procedure successfully equated the groups on overall $(\mathrm{R}+\mathrm{N})$ accuracy. The mean $(\mathrm{R}+\mathrm{N}$ ) overall accuracy scores were: schizophrenic, 52.2\% ( $\mathrm{SD}=2.34 \%$ ) ; bipolar, 52.8\% ( SD $=1.96 \%)$; normal control, 51.7\% ( $\mathrm{SD}=2.26 \%)$, F $(2,57)=1.16$, ns. Therefore, the potential artifactual effect of the $(\mathrm{R}+\mathrm{N})$ overall accuracy on the $(\mathrm{R}-\mathrm{N})$ difference score of facilitation should be minimal and should not affect their interpretation. The overall neutral plus unrelated $(\mathrm{N}+\mathrm{U})$ accuracy scores, which were not titrated, were: schizophrenic, 38.9\% ( SD = 4.51\%); bipolar, 46.9\% ( SD = 4.79\%) ; normal control, 44.9\% ( SD = 6.39\%), F $(2,57)=12.36, \mathrm{p}<.01$. Therefore, the psychometric artifact would tend to reduce the $(\mathrm{N}-\mathrm{U})$ inhibition score of the schizophrenic group more than the scores of the other groups, because the schizophrenic group was the farthest away from $50 \%$ accuracy on $(\mathrm{N}+\mathrm{U})$ overall accuracy. 
The effects of the three lists of items on facilitation and inhibition were examined. Neither the main effect for lists nor the Groups $\times$ List interaction for facilitation were significant, $F(2,51)=$ 1.58 and $\mathrm{F}(4,51)=0.69$, ns, respectively. Likewise, for inhibition, the main effect for lists and Groups $\times$ Lists interaction were not significant, $F(2,51)=1.42$ and $F(4,51)=1.02$, respectively. Therefore, the subsequent analyses disregard the effects of the lists.

The presence of overall facilitation was tested by a comparison of the grand mean related score with the grand mean neutral score, combining all the subjects into a single group. Similarly, the overall test for inhibition was the comparison of the grand mean unrelated score with the grand mean neutral score. Both comparisons were significant, $F(1,59)=65.28, \mathrm{p}<.001$, and $\mathrm{F}(1,59)$ $=23.33, \mathrm{p}<.01$, respectively. These results indicate that the procedures produced both semantic facilitation and inhibition.

The three groups were compared on facilitation and inhibition. The groups differed on facilitation, $F(2,57)=6.79, \mathrm{p}<.01$. Using the Newman-Keuls test to compare pairs of group means, the schizophrenic group was found to differ from both the control group, q $(3,57)=4.93$, $\mathrm{p}<.01$, and the bipolar group, $\mathrm{q}(2,57)=3.85, \mathrm{p}<.01$. However, the bipolar and control groups did not differ, $q(2,57)=0.89$. The three groups did not differ on inhibition, $F(2,57)=2.14$.

The differences between the groups on facilitation were not due to different levels of degradation. Mean degradation levels (percentage of pixels deactivated for each target word) were: schizophrenic, 70.69; bipolar, 69.00; and control, 71.41. These means did not differ, F (2, 57) $=1.01$.

The three groups were also compared on their absolute level of performance on the related, neutral, and unrelated component scores. The groups differed on the related items, F $(2,57)=$ $5.39, \mathrm{p}<.01$. Using the Newman-Keuls test to compare pairs of group means, the schizophrenic group was found to differ from both the control group, q $(3,57)=4.58, \mathrm{p}<.01$, and the bipolar group, $\mathrm{q}(2,57)=2.87, \mathrm{p}<.05$. However, the bipolar and control groups did not differ from one another, $\mathrm{q}(2,57)=1.53$. Likewise, the groups differed on the neutral items, although by necessity, in the opposite direction than on the related items, $F(2,57)=6.69, \mathrm{p}<.01$. Again, the schizophrenic group was found to differ from both the control group, q $(3,57)=6.69, \mathrm{p}<.01$. Again, the schizophrenic group was found to differ from both the control group, q $(3,57)=4.53$, $\mathrm{p}<.01$, and the bipolar group, $\mathrm{q}(2,57)=4.37, \mathrm{p}<.01$. However, the bipolar and control groups did not differ, $q(2,57)=0.03$. These findings demonstrate that the difference between groups on facilitation was not attributable to only one of the component scores. Nevertheless, the reader should be reminded that it is only the difference scores, not the component scores, that are meaningful. The groups also differed on their performance on the unrelated items, $\mathrm{F}(2,57)=$ 8.60, $\mathrm{p}<.001$. Using the Newman-Keuls test to compare pairs of groups, the schizophrenic group was found to differ from both the control group, $\mathrm{q}(2,57)=3.80, \mathrm{p}<.05$, and the bipolar group, $\mathrm{q}(3,57)=5.74, \mathrm{p}<.01$. Again, the bipolar and control groups did not differ, $\mathrm{q}(2,57)=$ 2.08. 
The groups were also compared on the number of trials that were scored as nonresponses. The Group $\times$ Conditions interaction was not significant, $F(4,114)=1.75$. However, the mean nonresponse rates, averaged across conditions, did differ between the groups: schizophrenic, 9.6\%; bipolar, 6.5\%; and control, 5.2\%; F $(2,57)=3.62$, $\mathrm{p}<.05$. Therefore, the main effect for facilitation was reanalyzed, with the trials scored as nonresponses eliminated from the analysis,

rather than scored as incorrect. The result was substantially identical to the initial finding in that the groups differed, $F(2,57)=6.21, p<.01$. Using the Newman-Keuls test to compare pairs of group means, the schizophrenic group was found to differ from both the control group, q $(3,57)$ $=4.84, \mathrm{p}<.01$, and the bipolar group, $\mathrm{q}(2,57)=3.39, \mathrm{p}<.05$. However, the bipolar and control groups did not differ, q $(2,57)=1.25$.

\section{Discussion}

The increased effects of semantic priming demonstrated by schizophrenic subjects is consistent with Maher's hypothesis that schizophrenics show heightened effects of semantic priming. The effects of overall accuracy as an artifact in the facilitation difference score were ruled out by the titration design. It is unclear whether the schizophrenics' heightened priming scores are accounted for by a stronger activation or a delayed activation of associates, a greater persistence of associates, or a defect in the inhibition of associates. The finding that schizophrenic subjects did not differ from control subjects on a measure of inhibition is consistent with the principle ( de Groot, 1984; de Groot et al., 1986; Posner \& Snyder, 1975) that processes other than automatic spreading activation are important for inhibition.

A possible problem with the future use of degradation of stimuli to titrate accuracy is that stimulus degradation might affect priming. Meyer, Schvaneveldt, and Ruddy (1975) studied the effects of stimulus degradation on semantic priming as measured by difference between latencies to primed and unprimed stimuli in a lexical decision task. They degraded stimuli by superimposing a grid of dots and found that degradation increased all of the response latencies as well as the difference between the latencies to the primed and unprimed target stimuli. Thus, their design did not make it possible to separate the effects of degradation per se from the effects of increased generalized slowness. As previously mentioned, a proportionate increase of the component latencies will tend to increase the difference between two latencies. Although the increase in overall latency in Meyer et al.'s study would tend to produce artifactually a heightened priming score, such as that reported for degradation, the possibility remains that degradation has a separate effect on priming. In any case, such effects would not account for the difference between schizophrenics and normal control subjects in the present study because the two groups did not differ significantly in level of stimulus degradation.

It is possible, because of the length of the interval between the priming stimulus and the subject's response, that strategic deployment of attention could have contributed to facilitation effects. However, the finding of heightened facilitation for schizophrenics is not reasonably attributable to the use of such attentional processing because schizophrenics are notoriously poor at directing 
their attention. Any attentional processing would tend to work against the hypothesis of increased facilitation by schizophrenics. The use of shorter stimulus-onset asynchrony intervals would minimize any attentional effects and would possibly increase the schizophrenic-normal difference found in the present study.

The finding that the schizophrenic subjects, but not the bipolar subjects, differed from the control subjects on facilitation raises the possibility that the priming task may tap a feature of associational processing that distinguishes schizophrenics from some other pathological groups. However, this finding may lack generality because other psychotic groups were not tested, the subjects received different types and dosages of psychotropic medications, and the schizophrenic and bipolar subjects may have had different degrees of disturbance. It was not possible in this study to separate the effects of group membership and medication because the majority of the schizophrenic subjects received neuroleptics, whereas the majority of the bipolar subjects received lithium carbonate. Furthermore, more than one third of the schizophrenic subjects received anticholinergics. These differing medications and dosages may have had disparate effects on the task performance. In addition, not all of the bipolar subjects had a history of psychotic symptoms. Future research should examine a more severely disturbed sample of bipolar subjects, as well as other pathological groups.

The use of counterbalanced lists of items, in which each target word appeared in each of the three conditions, conclusively ruled out differential responses to target-word characteristics in the three conditions as an interpretation of the results. However, the use of this procedure meant that different subjects within groups received different lists of items and ruled out the possibility of examining individual differences. Although the measure of facilitation yielded significant differences between the schizophrenic and the control groups, large individual differences occurred within the schizophrenic group. The range of facilitation was 3.1 to 34.4. These individual differences might have been due to the heterogeneity of the schizophrenic sample but might also reflect the use of different lists. Future studies should examine the correlates of individual differences in semantic priming among schizophrenics.

\section{References:}

American Psychiatric Association. ( 1980). Diagnostic and statistical manual of mental disorders ( 3rd ed.). Washington, DC: Author.

Antos, S. J. ( 1979). Processing facilitation in a lexical decision task. Journal of Experimental Psychology: Human Perception and Performance, 5, 527- 545.

Balota, D. A., \& Chumbley, J. I. ( 1984). Are lexical decisions a good measure of lexical access? The role of word frequency in the neglected decision stage. Journal of Experimental Psychology: Human Perception and Performance, 10, 340- 357. 
Bleuler, E. ( 1950). Dementia praecox or the group of schizophrenias (J.Zinkin, Trans.)New York: International Universities Press. ( Original work published 1911)

Chapman, L. J., \& Chapman, J. P. ( 1988). Artifactual and genuine relationships of lateral difference scores to overall accuracy in studies of laterality. Psychological Bulletin, 104, 127136.

Chapman, L. J., \& Chapman, J. P. ( 1989). Strategies for resolving the heterogeneity of schizophrenics and their relatives using cognitive measures. Journal of Abnormal Psychology, 98, 357-366.

de Groot, A. M. B. ( 1984). Primed lexical decision: Combined effects of the proportion of related prime-target pairs and the stimulus-on-set asynchrony of prime and target. Quarterly Journal of Experimental Psychology, 36A, 253- 280.

de Groot, A. M. B., Thomassen, A. J. W. M., \& Hudson, P. T. W. ( 1982). Associative facilitation of word recognition as measured from a neutral prime. Memory and Cognition, 10, 358-370.

de Groot, A. M. B., Thomassen, A. J. W. M., \& Hudson, P. T. W. ( 1986). Primed-lexical decision: The effect of varying the stimulus-onset asynchrony of prime and target. Acta Psychologica, 61, 17- 36.

Gjerde, P. F. ( 1983). Attentional capacity dysfunction and arousal in schizophrenia. Psychological Bulletin, 93, 57- 72.

Jonides, J., \& Mack, R. ( 1984). On the cost and benefit of cost and benefit analysis. Psychological Bulletin, 96, 29- 44.

Kucera, H., \& Francis, W. N. ( 1967). Computational analysis ofpresent day American English. Providence, RI: Brown University Press.

Maher, B. A. ( 1983). A tentative theory of schizophrenic utterance. In B.A. \& W B.Maher (Eds.), Progress in experimental personality research: Vol. 12. Psychopathology (pp. 1- 52). New York: Academic Press.

Manschreck, T. C., Maher, B. A., Milavetz, J. J., Ames, D., Weisstein, C. C., \& Schneyer, M. L. (1988). Semantic priming in thought disordered schizophrenic patients. Schizophrenia Research, $1,61-66$.

Massaro, D. W., Jones, R. D., Lipscomb, C., \& Scholz, R. ( 1978). Role of prior knowledge on naming and lexical decisions with good and poor stimulus. Journal of Experimental Psychology: Human Learning and Memory, 4, 498- 512. 
Meyer, D. E., \& Schvaneveldt, R. W. ( 1971). Facilitation in recognizing pairs of words: Evidence of a dependence between retrieval operations. Journal of Experimental Psychology, 90, 227- 234 .

Meyer, D. E., Schvaneveldt, R. W., \& Ruddy, M. G. ( 1975). Loci of contextual effects on visual word recognition. In P.Rabbitt \& S.Dornic (Eds.) , Attention and performance (vol. 5). New York: Academic Press.

Neely, J. H. ( 1977). Semantic priming and retrieval from lexical memory: Roles of inhibitionless spreading activation and limited capacity attention. Journal of Experimental Psychology: General, 106, 226- 254.

Nuechterlein, K. H. ( 1977). Reaction time and attention in schizophrenia: A critical evaluation of the data and theories. Schizophrenia Bulletin, 3, 373- 428.

Nuechterlein, K. H., \& Dawson, M. E. ( 1984). Information processing and attentional functioning in the developmental course of schizophrenic disorders. Schizophrenia Bulletin, 10, $160-203$.

Onifer, W. M. ( 1980). Associative intrusions in schizophrenic language Unpublished doctoral dissertation, Tufts University.

Posner, M. I., \& Snyder, C. R. R. ( 1975). Attention and cognitive control. In R. L.Solso (Ed.), Information processing and cognition: The Loyola symposium (pp. 55- 85). Hillsdale, NJ: Erlbaum.

Reed, A. V. ( 1979). Microcomputer display timing: Problems and solutions. Behavior Research Methods \& Instrumentation, 11, 572- 576.

Riegel, K. F., \& Riegel, R. M. ( 1961). Prediction of word-recognition thresholds on the basis of stimulus parameters. Language and Speech, 4, 157- 170.

Schvaneveldt, R. W., \& Meyer, D. E. ( 1973). Retrieval and comparison processes in semantic memory. In S.Kornblum (Ed.), Attention and performance (vol. 4) (pp. 394-410). New York: Academic Press. 